\title{
Comparison OfAzimuthal Ion Velocity Profiles Using Mach Probes, Time Delay Estimation, And Laser Induced Fluorescence In A Linear Plasma Device
}

S. Chakraborty Thakur

D. McCarren

T. Lee

N. Fedorczak

P. Manz

See next page for additional authors

Follow this and additional works at: https://researchrepository.wvu.edu/faculty_publications

\section{Digital Commons Citation}

Thakur, S. Chakraborty; McCarren, D.; Lee, T.; Fedorczak, N.; Manz, P.; Scime, E. E.; Tynan, G. R.; Xu, M.; and Yu, J., "Comparison Of Azimuthal Ion Velocity Profiles Using Mach Probes, Time Delay Estimation, And Laser Induced Fluorescence In A Linear Plasma Device" (2012). Faculty Scholarship. 491.

https://researchrepository.wvu.edu/faculty_publications/491 
Authors

S. Chakraborty Thakur, D. McCarren, T. Lee, N. Fedorczak, P. Manz, E. E. Scime, G. R. Tynan, M. Xu, and J. $\mathrm{Yu}$ 


\title{
Comparison of azimuthal ion velocity profiles using Mach probes, time delay estimation, and laser induced fluorescence in a linear plasma device ${ }^{\text {a) }}$
}

\author{
S. Chakraborty Thakur, ${ }^{1,2, b)}$ D. McCarren, ${ }^{3}$ T. Lee, ${ }^{2}$ N. Fedorczak, ${ }^{1,2}$ P. Manz, ${ }^{1,2}$ \\ E. E. Scime, ${ }^{3}$ G. R. Tynan, ${ }^{1,2}$ M. Xu, ${ }^{1,2}$ and J. Yu ${ }^{2}$ \\ ${ }^{1}$ Center for Momentum Transport and Flow Organization, University of California at San Diego, San Diego, \\ California 92093, USA \\ ${ }^{2}$ Center for Energy Research, University of California at San Diego, San Diego, California 92093, USA \\ ${ }^{3}$ Department of Physics, West Virginia University, Morgantown, West Virginia 26506, USA
}

(Presented 9 May 2012; received 7 May 2012; accepted 2 June 2012;

published online 9 July 2012)

\begin{abstract}
We compare measurements of radially sheared azimuthal plasma flow based on time delay estimation (TDE) between two spatially separated Langmuir probes, Mach probes and laser induced fluorescence (LIF). TDE measurements cannot distinguish between ion fluid velocities and phase velocities. TDE and Mach probes are perturbative, so we compare the results against LIF, a non-perturbative, spatially resolved diagnostic technique that provides direct measurements of the ion velocity distribution functions. The bulk ion flow is determined from the Doppler shift of the Argon absorption line at $668.6139 \mathrm{~nm}$. We compare results from all the three diagnostics, at various magnetic fields, which acts as a control knob for development of drift wave turbulence. We find that while Mach probes and LIF give similar profiles, TDE measurements typically overestimate the velocities and are also sensitive to the drift wave modes being investigated. (c) 2012 American Institute of Physics. [http://dx.doi.org/10.1063/1.4731005]
\end{abstract}

\section{INTRODUCTION}

The loss of particles and energy from the confinement regions of a magnetically confined fusion plasma is believed to be driven by turbulent drift wave fluctuations. Drift waves are one of the fundamental modes in magnetized plasmas and detailed reviews of both the theoretical ${ }^{1}$ and the experimental ${ }^{2}$ aspects are available in the literature. Drift waves can non-linearly drive poloidally and toroidally symmetric shear flows, called zonal flows, via the turbulent Reynolds stress. ${ }^{3}$ Zonal flows in turn lead to transport barriers, de-correlation of the turbulence and limit the absolute magnitude of the turbulence. ${ }^{3}$ The interaction of turbulence and zonal flows has developed into one of the most active research topics in the physics of fusion related magnetized plasmas. Experimental studies in linear magnetized devices are underway to understand the generation mechanism of zonal flows and the details of their interaction with drift wave turbulence. ${ }^{4-11}$

Previous studies ${ }^{12}$ in the Controlled Shear Decorrelation eXperiment (CSDX) have demonstrated a controlled transition to a turbulent state as the magnetic field $(\mathbf{B})$ is increased from $300 \mathrm{G}$ to $1000 \mathrm{G}$. With increasing $\mathbf{B}$, the plasma drift wave fluctuations evolve from narrow-band coherent perturbations to a state of weak turbulence characterized by broadened frequency and wave number spectra. At $\mathbf{B}=1000 \mathrm{G}$, narrow-band coherent mode-like drift wave fluctuations coexist with the more broadband turbulent fluctuations, hence

\footnotetext{
a) Contributed paper, published as part of the Proceedings of the 19th Topical Conference on High-Temperature Plasma Diagnostics, Monterey, California, May 2012.

b) Author to whom correspondence should be addressed. Electronic mail: saikat@ucsd.edu.
}

we call this a state of weak turbulence. At higher magnetic fields, studies in CSDX also show the presence of a turbulence driven azimuthally symmetric shear flow without any external sources of momentum input. ${ }^{4,5}$ Nonlinear energy transfer analyses ${ }^{7,13}$ have shown that for $\mathbf{B}=1000 \mathrm{G}$, the energy is transferred from the higher frequency $(f \sim 10 \mathrm{kHz}, m=3$ mode) turbulent fluctuations to the low frequency $(f<1 \mathrm{kHz}$, $m=0$ mode) azimuthally symmetric shear flow.

The above mentioned flow measurements were based on time delay estimation (TDE). The TDE technique has also been applied to density fluctuation measurements obtained by beam emission spectroscopy. ${ }^{14}$ It is however important to note that TDE measures the speed of propagation of fluctuations, which might differ from the actual advection of particles due to guiding center drifts. The fluctuations might not be perfect passive tracers of the background flow since diamagnetic drifts can introduce a phase velocity with respect to the guiding center drifts. ${ }^{1}$ TDE flows in CSDX, when compared with conventional Mach probe measurements ${ }^{15}$ at $\mathbf{B}=1000 \mathrm{G}$, are typically higher. Both Mach probes and TDE are perturbative. Moreover, interpretation of Mach probe data in unmagnetized or weakly magnetized (based on the ratio of the ion gyro-radius to the probe tip size) plasmas has its own complications. ${ }^{16}$ Laser induced fluorescence (LIF) ${ }^{17}$ is a non-perturbative, spatially resolved diagnostic technique used to obtain velocity distribution functions (VDFs) from which the absolute Doppler shifted velocity is determined. In this work we perform a comparative study of the three independent techniques of measuring the azimuthal velocity profiles as the magnetic field is systematically scanned from $400 \mathrm{G}$ (mode dominated) to $1000 \mathrm{G}$ (turbulence dominated) plasma. 


\section{EXPERIMENTAL APPARATUS}

The experiments are carried out in a cylindrical plasma device CSDX, whose details can be found elsewhere. ${ }^{12,18}$ For standard operating conditions, typical electron temperatures and plasma densities in CSDX, as measured by rf compensated, ${ }^{19}$ cylindrical Langmuir probes are $T_{e} \approx 3 \mathrm{eV}$ and $n \approx 10^{13} \mathrm{~cm}^{-3}$. Radial profiles of the plasma density, electron temperature, and floating potential as the magnetic field is scanned from low $(\mathbf{B}=400 \mathrm{G})$ to high $(\mathbf{B}=1000 \mathrm{G})$ fields are shown in Ref. 12. All data shown here are obtained at a distance of $75 \mathrm{~cm}$ downstream of the exit plane of the plasma source. All probe data were sampled at $1 \mathrm{MHz}$ for $2 \mathrm{~s}$. In the absence of strong electron temperature fluctuations, ion saturation current fluctuations (probe tips biased to $-30 \mathrm{~V}$ ) are interpreted as density fluctuations and floating potential fluctuations are a proxy for plasma potential fluctuations.

\section{DIAGNOSTICS}

For TDE measurements, density fluctuations are sampled at a rate $1 / \Delta t$, and the peak time lag $\tau_{p}$ is calculated between two spatially separated probes based on the peak of the cross correlation

$$
R_{\Delta x}(\tau)=\frac{1}{T} \int_{t-T / 2}^{t+T / 2} d t^{\prime} n\left(x, t^{\prime}\right) n\left(x+\Delta x, t^{\prime}+\tau\right),
$$

where $n$ is the measured signal and $T$ is the duration over which each cross correlation is calculated. The TDE velocity is calculated from the peak time lag and the known probe separation distance $\Delta x$. By moving the time window $\mathrm{T}$ and repeating the calculations, an ensemble of several different $V_{T D E}=\Delta x / \tau_{p}$ can be calculated. The mean and statistical variation in $V_{T D E}$ is obtained by averaging over $N$ subwindows. The range of velocities measured by TDE is limited by the discrete sampling during data acquisition. If $T$ is the length of the time window under consideration, then $\left(V_{T D E}\right)_{\min }$ $=\Delta x /\left(\tau_{p}\right)_{\max }$ where $\left(\tau_{p}\right)_{\max }=T / 2$. Discrete sampling also sets a maximum velocity that can be inferred by TDE, $\left(V_{T D E}\right)_{\max }=\Delta x / \Delta t$ such that the fluctuations do not move the distance $\Delta x$ in a time smaller than $\Delta t$. Moreover, for studying turbulent plasma, it is important that the turbulence decorrelation timescale $\tau_{\text {decor }}$ should be longer than the time interval $T$ to ensure that the flow is approximately steady within $T$, so that the Taylor frozen-flow hypothesis is not violated. We used three pairs of probe tips, ${ }^{6}$ placed $1.5 \mathrm{~mm}$ apart with $\Delta x$ $=2.54 \mathrm{~mm}$ each, to obtain three simultaneous realizations of the azimuthal velocity, at three locations within $5 \mathrm{~mm}$.

A four tip Mach probe is used to measure the bulk plasma velocity. The tips, placed symmetrically about the probe axis and separated by insulators, are biased negatively to collect ion saturation currents. The flows are interpreted based on the ratio $\left(R_{M}\right)$ of the ion saturation currents collected by $180^{\circ}$ opposite probe tips. $R_{M}$ is measured as a function of $\theta$, the angle between the magnetic field and the line connecting the $180^{\circ}$ opposite probes. The measurement are then fitted to the equation $^{20}$

$R_{M}=\exp \left[\mathrm{K} \sin (\Delta \alpha) / \Delta \alpha\left(M_{\mathrm{par}} \cos \theta+M_{\mathrm{perp}} \sin \theta\right)\right]$, where $\Delta \alpha$ is the acceptance angle of each probe tip $\left(\sim 50^{\circ}\right.$ in our probe design), $M_{\text {par }}$ and $M_{\text {perp }}$ are used as fitting parameters and using Hutchinson's model ${ }^{21}$ of ion collection, we have $\mathrm{K}=1.34$. This model of Mach probe analysis requires the probe to be rotated by $360^{\circ}$ for each radial location. This method has been shown to be reliable for various Mach probe geometries in weakly magnetized plasma. ${ }^{20}$

For LIF measurements, ${ }^{17}$ a Toptica TA 100 tunable diode laser was used. The laser had a line width of $\sim 1 \mathrm{MHz}$ and a mode hop free tuning range of $\sim 30 \mathrm{GHz}$. For these measurements, the laser was tuned to $668.6138 \mathrm{~nm}$, corresponding to $3 \mathrm{~d}^{4} \mathrm{~F}_{7 / 2}$ to $4 \mathrm{p}^{4} \mathrm{D}_{5 / 2}$ level transition in Ar II. A $10 \%$ beam-splitter was used to sample the beam and was coupled into a Bristol Instruments 621-VIS wavemeter for real-time wavelength monitoring. The wavelength was measured to an accuracy of $\pm 0.0001 \mathrm{~nm}$. The error in the measured velocity due to this instrumental error is $\sim 60 \mathrm{~m} / \mathrm{s}$. The other $90 \%$ of the laser light was mechanically chopped at a few $\mathrm{kHz}$ and transported to the plasma chamber by beam steering mirrors.

The fluorescent emission at $442.60 \mathrm{~nm}$ from the decay of $4 p^{4} D_{5 / 2}$ state to the $4 s^{4} P_{3 / 2}$ state was coupled into a photomultiplier tube through a $1 \mathrm{~nm}$ wide filter and recorded by a lockin-amplifier. The intensity of the fluorescent emission from the excited state as a function of laser frequency is a direct measurement of the ion VDF in the region where the injected beam overlaps the collection volume. In this experiment, the spatial resolution of the LIF measurements was $\sim 3 \mathrm{~mm}$. To determine the Doppler broadened ion temperature and the bulk azimuthal velocity, a nonlinear least squares fitting routine fits the measured ion VDF with a drifting Maxwellian distribution of the form

$$
I_{R}(v)=I_{R}\left(v_{o}\right) e^{-m_{i} c^{2}\left(v-v_{o}\right)^{2} / 2 k T_{i} v_{o}^{2}},
$$

where $v_{o}$ is the rest frame frequency of the absorption line, $m_{i}$ the ion mass, and $T_{i}$ the ion temperature. The shift of the peak of the distribution from the rest frame absorption frequency line gives the bulk perpendicular poloidal ion speed.

\section{RESULTS AND DISCUSSIONS}

In Figs. 1(a) and 1(b), we show radial profiles of the azimuthal velocity as measured by TDE, Mach probe, and LIF for $\mathbf{B}=400 \mathrm{G}$ and $\mathbf{B}=600 \mathrm{G}$. For these smaller magnetic fields, the plasma is dominated by wave like modes. ${ }^{12}$ We do not observe strong plasma rotation or velocity shear. Figs. 1(c) and 1(d) show velocity measurements for larger magnetic fields, $\mathbf{B}=800 \mathrm{G}$ and $\mathbf{B}=1000 \mathrm{G}$, the weak turbulence regime. Here, the velocities are peaked and there is a strong radially sheared plasma flow. The positive velocity is in the electron diamagnetic drift direction. All three diagnostics show similar trends, i.e., both the peak velocity and the strength of the velocity shear (the radial gradient in the azimuthal velocity) increase with increasing magnetic field, concurrent with an increase in the turbulent fluctuation levels.

Mach probe and the LIF measure the bulk plasma flow, so the velocity profiles are similar for all values of magnetic field. LIF measurements are consistent with calculated $\boldsymbol{E} \times \boldsymbol{B}$ and ion diamagnetic drifts. LIF-derived flows have a slightly lower peak velocity, which occurs at a smaller plasma 

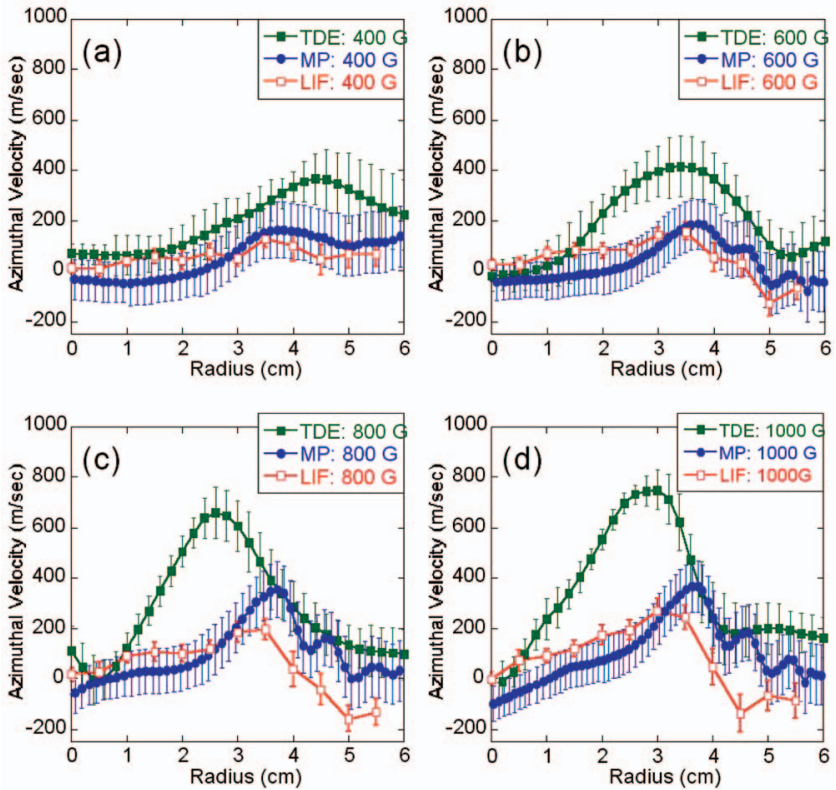

FIG. 1. Radial profiles of the azimuthal velocity for (a) $400 \mathrm{G}$, (b) $600 \mathrm{G}$, (c) $800 \mathrm{G}$, and (d) $1000 \mathrm{G}$, from TDE (green solid squares), Mach probe (blue solid circles), and LIF (red empty squares) measurements.

radius than the peak measured by the Mach probe. However, TDE cannot distinguish between the bulk plasma flow and the phase velocity of the waves propagating in the plasma and hence give higher velocities compared to LIF and the Mach probe. The difference in the magnitudes grows with $\mathbf{B}$, correlating with increased fluctuation at higher $\mathbf{B} .{ }^{12}$ Also noticeable is a shift of the radial location of the peak in the TDE-derived flow. There are two possible explanations for the shift. The radial location of the maxima of the density and potential fluctuations depends on B (Ref. 12) and is the same as where the TDE flows peak. As $\mathbf{B}$ increases, the pres-

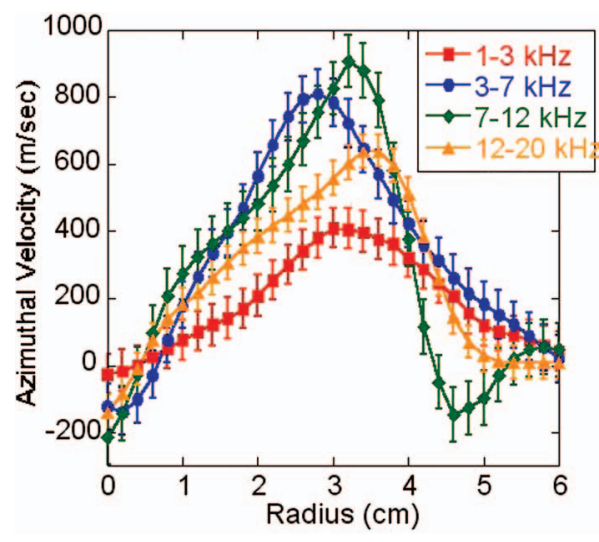

FIG. 2. Radial profiles of the azimuthal velocity (using TDE) for different modes at $\mathbf{B}=1000 \mathrm{G}$. Red squares are $m=1(1<f<3 \mathrm{kHz})$, blue circles are $m=2(3<f<7 \mathrm{kHz})$, green diamonds are $m=3$ modes $(7<f$ $<12 \mathrm{kHz}$ ), and orange triangles represent the background turbulence $(12<f<20 \mathrm{kHz})$. sure gradients not only increase in magnitude, but also move inwards, similar to the peak of the TDE velocities. The corresponding electron diamagnetic drifts might have a role in explaining this discrepancy. Also as $\mathbf{B}$ changes, the dominant frequencies and modes in the plasma change. ${ }^{12,13}$ While Mach probe and LIF are time averaged measurements, TDE is averaged over the frequencies used in calculating cross correlations of the phase velocity of fluctuations. In Fig. 2 we show the TDE velocities at which the individual modes propagate in the plasma, for $\mathbf{B}=1000 \mathrm{G}$. Notice that both the peak velocities and the radial location of the peaks differ with the mode number. Fast camera imaging data ${ }^{6,8}$ show that in the turbulent cases, all the modes are not equally represented in the plasma. There is a slow time evolution over a few milliseconds when the $m=3$ mode nonlinearly loses the energy to the background shear flow and enhances it. Then there is a rapid collapse phase (few hundreds of microseconds) when the shear strength goes down and blobs are ejected radially outwards. During the collapse phase and the blob ejection, $m=1$ mode dominates. ${ }^{22}$ It is likely that these different modes get advected differently and should be weighed accordingly to get time averaged TDE velocity values for proper comparison with the LIF and Mach probe measurements. We find that the $m=1$ velocity profiles closely resemble that of the LIF and Mach probe, for all values of $\mathbf{B}$.

In conclusion, we find that for a wide range of $\mathbf{B}$ in CSDX, LIF and Mach probes give similar azimuthal velocity profiles. TDE-derived flows have similar magnetic field dependency, but further analysis is required to isolate the plasma flow component. But unlike Mach probes or LIF, TDE can possibly give information of individual modes in the plasma and efforts are on to quantify them and study their effects on turbulence.

\footnotetext{
${ }^{1}$ W. Horton, Rev. Mod. Phys. 71, 735 (1999).

${ }^{2}$ G. R. Tynan, A. Fujisawa, and G. Mckee, Plasma Phys. Controlled Fusion 51, 113001 (2009).

${ }^{3}$ P. H. Diamond, S.-I. Itoh, K. Itoh, and T. S. Hahm, Plasma Phys. Controlled Fusion 47, R35 (2005).

${ }^{4}$ C. Holland et al., Phys. Rev. Lett. 96, 195002 (2006).

${ }^{5}$ G. R. Tynan et al., Plasma Phys. Controlled Fusion 48, S51 (2006).

${ }^{6}$ Z. Yan et al., Phys. Plasmas 15, 092309 (2008).

${ }^{7}$ M. Xu et al., Phys. Plasmas 17, 032311 (2010).

${ }^{8}$ Z. Yan et al., Phys. Plasmas 17, 032302 (2010).

${ }^{9}$ Y. Nagashima et al., Phys. Plasmas 16, 020706 (2009).

${ }^{10} \mathrm{~T}$. Windisch, O. Grulke, and T. Klinger, J. Nucl. Mater. 390, 395 (2009).

${ }^{11}$ T. A. Carter and J. E. Maggs, Phys. Plasmas 16, 012304 (2009).

${ }^{12}$ M. J. Burin et al., Phys. Plasmas 12, 052320 (2005).

${ }^{13}$ P. Manz, M. Xu, S. C. Thakur, and G. R. Tynan, Plasma Phys. Controlled Fusion 53, 095001 (2011).

${ }^{14}$ G. R. Mckee et al., Phys. Plasmas 10, 1712 (2003).

${ }^{15}$ J. H. Yu et al., J. Nucl. Mater. 363-365, 728 (2007).

${ }^{16}$ I. H. Hutchinson, Phys. Plasmas 9, 1832 (2002).

${ }^{17}$ A. M. Keesee, R. Boivin, and E. E. Scime, Rev. Sci. Instrum. 75, 4091 (2004).

${ }^{18}$ G. R. Tynan et al., J. Vac. Sci. Technol. A 15, 2885 (1997).

${ }^{19}$ I. D. Sudit and F. F. Chen, Plasma Sources Sci. Technol. 3, 162 (1994).

${ }^{20}$ T. Shikama et al., Phys. Plasmas 12, 044504 (2005).

${ }^{21}$ I. H. Hutchinson, Plasma Phys. Controlled Fusion 47, 71 (2005).

${ }^{22}$ P. Manz et al., Phys. Rev. Lett. 107, 195004 (2011).
} 